\title{
Relativistic High-Current Electron-Beam Stopping-Power Characterization in Solids and Plasmas: Collisional Versus Resistive Effects
}

\author{
B. Vauzour, ${ }^{1}$ J. J. Santos, ${ }^{1, *}$ A. Debayle, ${ }^{2}$ S. Hulin, ${ }^{1}$ H.-P. Schlenvoigt,${ }^{3}$ X. Vaisseau, ${ }^{1}$ D. Batani,,${ }^{1,4}$ S. D. Baton, ${ }^{3}$ \\ J. J. Honrubia, ${ }^{2}$ Ph. Nicolaï, ${ }^{1}$ F. N. Beg, ${ }^{5}$ R. Benocci, ${ }^{4}$ S. Chawla,${ }^{5}$ M. Coury,${ }^{6}$ F. Dorchies, ${ }^{1}$ C. Fourment, ${ }^{1}$ E. d'Humières, ${ }^{1}$ \\ L. C. Jarrot, ${ }^{5}$ P. McKenna, ${ }^{6}$ Y. J. Rhee,${ }^{7}$ V. T. Tikhonchuk, ${ }^{1}$ L. Volpe, ${ }^{4}$ and V. Yahia ${ }^{3}$ \\ ${ }^{1}$ Univ. Bordeaux, CNRS, CEA, CELIA (Centre Lasers Intenses et Applications), UMR 5107, F-33405 Talence, France \\ ${ }^{2}$ ETSI Aeronáuticos, Universidad Politécnica de Madrid, Madrid, Spain \\ ${ }^{3}$ LULI, Ecole Polytechnique CNRS/CEA/UPMC, 91128 Palaiseau Cedex, France \\ ${ }^{4}$ Dipartimento di Fisica, Università di Milano-Bicocca, Milano 20126, Italy \\ ${ }^{5}$ University of California, San Diego, La Jolla, California 92093, USA \\ ${ }^{6}$ SUPA, Department of Physics, University of Strathclyde, Glasgow G4 ONG, United Kingdom \\ ${ }^{7}$ Korea Atomic Energy Research Institute (KAERI), Daejon 305-600, Korea
}

(Received 10 September 2012; published 18 December 2012)

\begin{abstract}
We present experimental and numerical results on intense-laser-pulse-produced fast electron beams transport through aluminum samples, either solid or compressed and heated by laser-induced planar shock propagation. Thanks to absolute $K_{\alpha}$ yield measurements and its very good agreement with results from numerical simulations, we quantify the collisional and resistive fast electron stopping powers: for electron current densities of $\approx 8 \times 10^{10} \mathrm{~A} / \mathrm{cm}^{2}$ they reach $1.5 \mathrm{keV} / \mu \mathrm{m}$ and $0.8 \mathrm{keV} / \mu \mathrm{m}$, respectively. For higher current densities up to $10^{12} \mathrm{~A} / \mathrm{cm}^{2}$, numerical simulations show resistive and collisional energy losses at comparable levels. Analytical estimations predict the resistive stopping power will be kept on the level of $1 \mathrm{keV} / \mu \mathrm{m}$ for electron current densities of $10^{14} \mathrm{~A} / \mathrm{cm}^{2}$, representative of the full-scale conditions in the fast ignition of inertially confined fusion targets.
\end{abstract}

PACS numbers: 52.50.-b, 52.38.Kd, 52.65.-y, 52.70.La

In the fast ignition (FI) scheme of inertial confinement fusion, a relativistic electron beam (REB) heats the compressed core and ignites the fusion reactions in a capsule of deuterium and tritium [1]. This REB is generated at the critical density surface, or at the cone tip of a cone-embedded imploded capsule [2] by a high-intensity $\left(\approx 10^{20} \mathrm{~W} / \mathrm{cm}^{2}\right)$ and high-energy $(\sim 100 \mathrm{~kJ})$ laser. The REB source has a total kinetic energy $\lesssim 40 \%$ of the laser energy [3-5] and a mean kinetic energy of 1-2 MeV (to provide an efficient coupling to the dense core). The REB transports energy from the generation region (with density and temperature in the level of a few $\mathrm{g} / \mathrm{cm}^{3}$ and a few eV, respectively) to the high-density $\left(\sim 400 \mathrm{~g} / \mathrm{cm}^{3}\right)$ and hightemperature $(\sim 300 \mathrm{eV})$ core, where it must deliver a minimum of $20 \mathrm{~kJ}$ to heat the fuel to thermonuclear temperatures $(\sim 5-10 \mathrm{keV})$ [6]. The energy transport efficiency can be limited by such physical processes as collisional or collective energy loss [7], divergence [8,9], filamentation [10-12], etc. The energy losses over the highly inhomogeneous electron transport zone should be accurately predicted for a successful full-scale FI design. In particular, the REB stopping power should be limited to a few $\mathrm{keV} / \mu \mathrm{m}$ over the $\sim 100 \mu \mathrm{m}$ standing-off distance between the REB source and the imploded core.

The work presented here aims at characterizing the REB stopping power in dense media in underscaled experimental conditions. The measurements are used to benchmark a REB transport code. The tested transport media, ranging from solid to warm dense matter, are much denser than the injected REB, being reasonable to assume an efficient neutralization of the injected current $\left(\vec{j}_{h}\right)$ by a counterstreaming current $\left(\vec{j}_{e}\right)$ of background thermal electrons $\left(\vec{j}_{h} \approx-\vec{j}_{e}\right)$. Under these conditions, the numerical description of the REB transport often uses the so-called hybrid approach, where the incident and weakly collisional electrons are modeled kinetically and the highly collisional return current is described as an inertialess fluid $[10,13,14]$.

Most of the REB transport experiments carried out up to now have used solid targets $[8,15,16]$. Only a few entered in the warm dense matter regime driving the targets by shock compression in planar [17-20] or cylindrical [21,22] geometries. For the sake of a precise stopping power characterization as a function only of the target material (density, temperature, resistivity, etc.) and independent of its particular geometry, we preferred a planar compression geometry with the REB injection in the opposite direction to the compressing shock. Aluminum samples of variable thickness, embedded in a multilayer foil-target structure, were shock compressed by a factor of $\sim 2$. The achieved temperatures were $\approx 2-3 \mathrm{eV}$, close to the material Fermi temperature $(\sim 11 \mathrm{eV})$, significantly changing its resistivity compared to cold samples, to which the results of REB transport are here systematically confronted. While in previous experiments the total electron beam energy losses were measured without distinction between resistive and collisional mechanisms, we show in this Letter, that we 
have been able to quantify separately the contributions of the two effects and their respective dependence on the electron beam current density. The results are relevant for the FI in the sense that one highlights the importance of resistive effects on REB energy losses, which are highly dependent on the temperature of the background medium. Indeed, our results, supported by numerical simulations, show that the total REB stopping power is higher in compressed samples due to an increase of both the material density and resistivity.

The experiment was carried out at LULI laboratory at Ecole Polytechnique (France) using the PICO2000 facility with a dual-laser beam configuration, a long pulse (LP) beam to drive the target compression, and a short pulse (SP) beam to generate the fast electrons (see Fig. 1). We used $5 \times 5 \mathrm{~mm}^{2}$ surface multilayer foil targets including an aluminum (Al) sample of variable thickness (10, 20, 40, or $60 \mu \mathrm{m}$ ), used as the propagation layer to study REB transport. The samples were coated on both sides by different $K$-shell fluorescence tracer layers: a $5 \mu \mathrm{m}$ silver $(\mathrm{Ag})$ layer at the front side used to characterize the fast electrons source and two successive $10 \mu \mathrm{m}$ layers of tin (Sn) and copper $(\mathrm{Cu})$, at the rear side, to characterize the fast electrons that have crossed the Al samples. On the rear surface a $15 \mu \mathrm{m}$ polypropylene (PP) layer was added as an ablator for the LP beam, improving the compression quality and also preventing direct laser damage or heating of the $\mathrm{Cu}$ and $\mathrm{Sn}$ tracer layers. The REB was generated by irradiating the front side of the targets, a $5 \mu \mathrm{m} \mathrm{Al} \mathrm{layer}$ coating the Ag layer, with the SP laser beam, of $1.5 \mathrm{ps}$ full width at half maximum duration. Its Gaussian focal spot of $10 \mu \mathrm{m}$ diameter (full width at half maximum) contained $25 \%$ of $35 \mathrm{~J}$, corresponding to a maximum intensity on target of $3 \times 10^{19} \mathrm{~W} / \mathrm{cm}^{2}$. The SP laser pedestal intensity, due to the amplified spontaneous emission, was evaluated to $10^{12} \mathrm{~W} / \mathrm{cm}^{2}$ approximately $1.1 \mathrm{~ns}$ before the main pulse [19]. The LP laser beam had an intensity of $3 \times 10^{13} \mathrm{~W} / \mathrm{cm}^{2}$ at $0.53 \mu \mathrm{m}$ wavelength. A duration of $4.5 \mathrm{~ns}$ (flattop time profile) was chosen to get a longitudinal homogeneous compression over the thickest samples. The large flattop focal spot (400 $\mu \mathrm{m}$ diameter), obtained by coupling a phase zone plate with the focusing lens, created a homogeneous compressed area much larger than the REB radius.

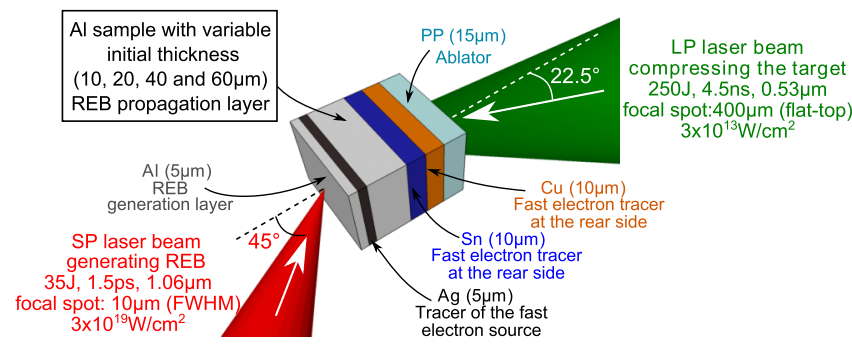

FIG. 1 (color online). Schematic of the target and of the laser beams setup.
The compressing shock speed in the Al sample was evaluated from streaked optical pyrometry of the shock breakout on the front side of specifically dedicated targets without the front $\mathrm{Al}$ and $\mathrm{Ag}$ layers. These measurements benchmarked 2D radiative-hydrodynamic simulations of the target compression using the code CHIC [23], and these allowed to specify the delays between LP and SP target irradiation for two conditions: when the shock just reached the $\mathrm{Sn} / \mathrm{Al}$ interface (short delay, the same for every sample thickness) and when the Al sample was almost entirely compressed (long delay, variable according to the sample thickness), corresponding, respectively, to study the REB transport in cold-solid $\left(T_{e}=0.03 \mathrm{eV}, \rho_{0}=2.7 \mathrm{~g} / \mathrm{cm}^{3}\right)$ or in warm-dense $\mathrm{Al}\left(T_{e} \sim 2-3 \mathrm{eV}, \rho \sim 2 \rho_{0}\right)$. The typical density and temperature on-axis profiles obtained from the simulations are shown in Fig. 2 for a $60 \mu \mathrm{m} \mathrm{Al}$-sample target, either solid (left) or compressed (right panel). Note that at the target's front side both the Al-electrongeneration layer and the Ag-electron source tracer are in the same conditions for the two case studies of the $\mathrm{Al}$ sample. Also note that the rear side (i.e., the $\mathrm{Sn}, \mathrm{Cu}$, and PP layers) of the solid-sample targets is also irradiated by the LP beam in order to create rear-side $K$-shell tracer conditions similar to the case of the driven samples. More particularly, this generates a long PP plasma tail where the background electron density is greater than the REB density over an extra $\sim 100 \mu \mathrm{m}$ beyond the $\mathrm{Cu}$ layer: the fast electrons are confined far beyond the $K$-shell tracers [24], allowing us to quantify the REB energy losses on the samples from only one REB transit.

The $\mathrm{Al}$ sample is compressed to roughly twice its initial density and its length diminished by the same factor, upon shock propagation. Consequently, its areal density $\rho L_{\mathrm{Al}}$ along the REB propagation axis remains constant. As the electron collisional stopping power is proportional to the density of the crossed material, one can fairly expect

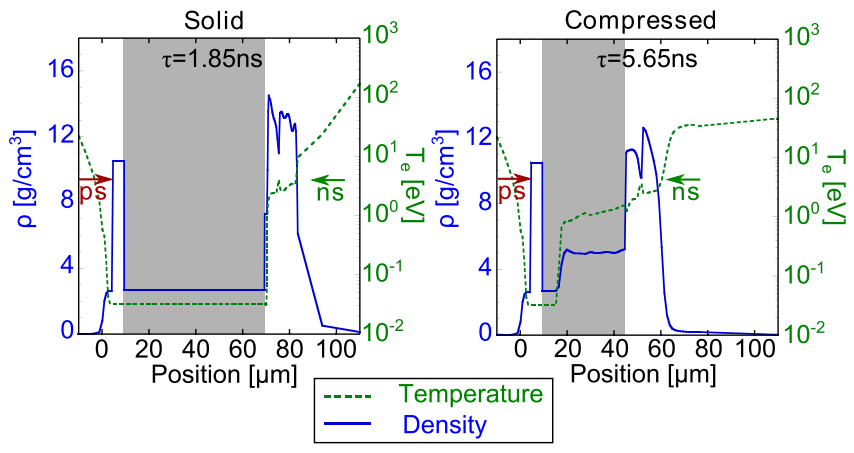

FIG. 2 (color online). On-axis density (blue solid lines) and temperature (green dashed lines) profiles obtained by 2D CHIC hydrodynamic simulations of a $60 \mu \mathrm{m}$ thick $\mathrm{Al}$ sample target (background gray area). The left and right panels represent the solid and compressed cases, respectively, just before the fast electron beam generation. The red and green arrows indicate the directions of the SP and LP laser beams, respectively. 
that the collisional energy losses integrated along the Al sample are the same for a given initial thickness, in both compressed and solid Al. This allows us to experimentally observe the importance of resistive energy losses on the REB propagation. Indeed, the resistive losses are not constant with the compression as they increase with the resistivity $\eta$ of the Al sample $\left(\eta_{\text {solid }} \sim 3 \times 10^{-8} \Omega \mathrm{m} \rightarrow\right.$ $\left.\eta_{\text {compressed }} \sim 5 \times 10^{-7} \Omega \mathrm{m}[25,26]\right)$ due to the temperature raise to $\approx 2 \mathrm{eV}$ (close to the Fermi temperature) where the resistivity is almost at its maximum and mostly governed by the electron-electron collisions.

In order to study the REB propagation inside the $\mathrm{Al}$ samples, $K_{\alpha}$ radiations, emitted at the fast electron passage through the tracer layers, were collected by several diagnostics. The REB geometry was measured using a $2 \mathrm{D}$ $\mathrm{Cu}-K_{\alpha}$ imaging technique [27], showing a $22 \pm 6^{\circ}$ cone half-angle divergence independent of the sample state. These results are reported elsewhere [28]. Fast electron energy losses were investigated with the help of a Cauchois-type hard-x-ray spectrometer [29] using a transmission curved quartz crystal $(2 d=0.6884 \mathrm{~nm})$ measuring the absolute rear $\mathrm{Sn}(\sim 25 \mathrm{keV})$ and front $\mathrm{Ag}-K_{\alpha}$ $(\sim 22 \mathrm{keV})$ yields.

The experimental results are compared to simulations of REB transport. The REB source parameters are estimated with the help of 2D particle-in-cell simulations using the code PICLS [30], set up to model the interaction of the SP laser with the target front-Al-layer. The profile of the preplasma, caused by the laser amplified spontaneous emission pedestal, was measured by side-on interferometry [19] and calculated with the CHIC code (see Fig. 2). The temporal profile of the laser pulse is assumed to be Gaussian shaped with a peak intensity set to $3 \times 10^{19} \mathrm{~W} / \mathrm{cm}^{2}$. Because of computing limitations, the laser pulse duration is reduced to $0.5 \mathrm{ps}$ (full width at half maximum). The fast electron source parameters are extracted at $\approx 1.5 \mu \mathrm{m}$ beyond the absorption region, located at $\approx 4.5 \mu \mathrm{m}$ from the initial target surface position. The measured kinetic energy distribution function is temporally averaged over the entire simulation time and fitted over the range $0.01<E<20 \mathrm{MeV}$ by the following normalized function:

$$
f(E)= \begin{cases}\left(\frac{E_{0}}{E}\right)^{\alpha} & \text { if } 0.01<E<5 \mathrm{MeV} \\ N \exp \left(-\frac{E}{T}\right) & \text { if } 5 \leq E<20 \mathrm{MeV}\end{cases}
$$

where $E_{0}=1.7 \mathrm{keV}, \alpha=1.6, T=3 \mathrm{MeV}$, and $N=$ $1 \times 10^{-5}$ are the fitting parameters. This function is plotted on Fig. 3 (red solid line) and compared to the distribution function extracted directly from the particle-in-cell (PIC) simulations (gray solid line). Note that the low-energy part of $f(E)$ corresponds to a power-law function with a decreasing trend given by the parameter $\alpha$. This provides a better fit to the distribution than the commonly used

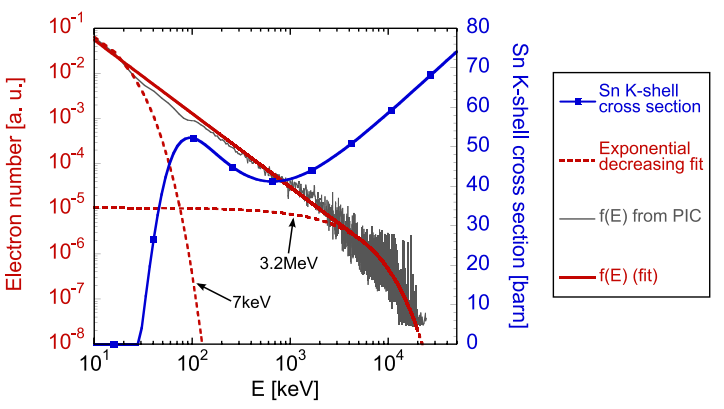

FIG. 3 (color online). Simulated (PIC) distribution function (gray line) and its associated fits: the red solid line corresponds to the fit $f(E)$ given by (1), and the two red dashed lines to the usual exponential decreasing fits. The blue solid line with squares represents the $\mathrm{Sn} K$-shell cross section.

Maxwellian functions (red dashed lines), especially in the energy range $30 \mathrm{keV}-5 \mathrm{MeV}$, where the $\mathrm{Ag}$ and $\mathrm{Sn}$ $K$-shell cross sections [31] (blue solid line with squares) are higher. The power law distribution function results in a good reproduction of the absolute $K_{\alpha}$ yields obtained experimentally, as described below. The angular distribution of the fast electron beam is fitted by the function $f(\theta)=\exp \left[-\left(\theta-\theta_{r}\right)^{2} / \Delta \theta_{0}^{2}\right]$ [9], with the dispersion angle $\Delta \theta_{0}=55^{\circ}$ and the mean radial angle $\theta_{r}=$ $\arctan \left[\tan \left(30^{\circ}\right) r / r_{0}\right]$, where $r_{0}$ is the initial REB radius. Finally, the laser-to-fast electron conversion efficiency, calculated by temporally and spatially integrating the total energy transported by the fast electron beam, is estimated to $30 \pm 10 \%$ for $0.01<E<20 \mathrm{MeV}$.

These REB source parameters are used as input parameters in the hybrid code [14] simulating the REB transport through the entire different targets having either compressed or solid Al samples. The targets' density and temperature are extracted from the hydrodynamic simulations at the REB injection time. The total simulation time is set to 8 ps with temporal and spatial resolutions of $1 \mathrm{fs}$ and $0.5 \mu \mathrm{m}$, respectively. Collisional and ionization processes are taken into account. The electrical resistivity of each material is computed using the classical Drude model: $\eta=m_{e} \nu / e^{2} n_{e}$, where $e$ and $m_{e}$ are the electron charge and rest mass, respectively, $n_{e}$ is the background electron density, and $\nu$ is the electron collision frequency given by the Eidmann-Chimier model $[25,26]$, which accounts for the contribution of the electron-phonon, electron-electron, and electron-ion collisions, according to the background density and temperature. A $K_{\alpha}$ emission module, based on the model of Ref. [31], is used to calculate the $K_{\alpha}$ signal yields and size for comparison with experiment.

Results from the hard-x-ray spectrometer are presented in Fig. 4 (solid symbols). The absolute $\mathrm{Sn}-K_{\alpha}$ yields are plotted on the left panel, showing a fairly good agreement between experimental (full symbols) and numerical (empty symbols) results, where the laser-to-fast electron 

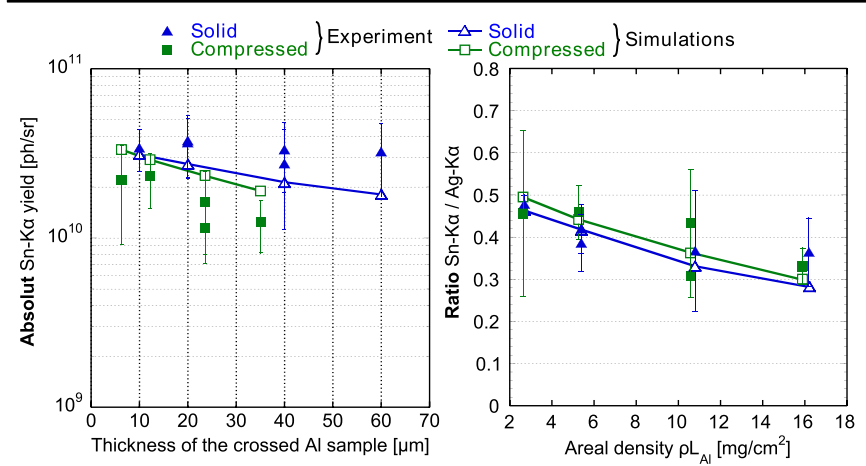

FIG. 4 (color online). (left) Comparison of the experimental and simulated evolution of the $\mathrm{Sn}-K_{\alpha}$ absolute yields as a function of the thickness of the crossed Al sample. (right) Comparison of the experimental and simulated evolution of the $\mathrm{Sn}-K_{\alpha}: \mathrm{Ag}-K_{\alpha}$ absolute yields ratio as a function of $\mathrm{Al}$ sample areal density.

energy conversion efficiency is set to $40 \%$ (value better fitting the absolute $\mathrm{Ag}$ and $\mathrm{Sn}-K_{\alpha}$ yields). On the right panel, the Sn- $K_{\alpha}: \mathrm{Ag}-K_{\alpha}$ yields ratio is plotted as a function of $\rho L_{\mathrm{Al}}$. This ratio allows the overcoming of any shotto-shot variations of the fast electron source and thus can be associated with the energy fraction of the fast electron population that has crossed the $\mathrm{Al}$ sample. The observed decreasing trends, for both compressed and solid targets, are a signature of a progressive energy loss of the fast electron beam as it propagates deeper inside the sample. Nonetheless, there is no striking difference between solid (in blue) and compressed (in green) samples as a function of $\rho L_{\mathrm{Al}}$, meaning that resistive energy losses are possibly too weak, in our interaction conditions, to be experimentally observable. This also means that the absolute $\mathrm{Sn}-K_{\alpha}$ yield variation against thickness (left panel in Fig. 4), with a higher decrease rate in the case of the compressed samples, is mainly due to a higher collisional rate associated with the sample density rise.

The REB energy losses in the $\mathrm{Al}$ samples are computed with the hybrid code and presented in Fig. 5. Collisional and resistive energy losses are represented by green squares and orange triangles, respectively. They were calculated for three laser energies 35,70 , and $350 \mathrm{~J}$, assuming a conversion efficiency of $40 \%$. The values labeling the different curves are the REB current densities at the sample front surface averaged over the beam radius and over time. The collisional losses are greater in compressed samples (dashed lines), due to the density rise (they are similar in solid and compressed targets of the same areal density). The lower current case (dark gray background area), corresponding to the parameters of the present experiment (same simulations as those giving results of Fig. 4), shows that the resistive losses are also greater in compressed compared to solid samples, resulting from a higher initial resistivity of the driven Al. Although the fast electron collisions are the predominant energy loss mechanism for

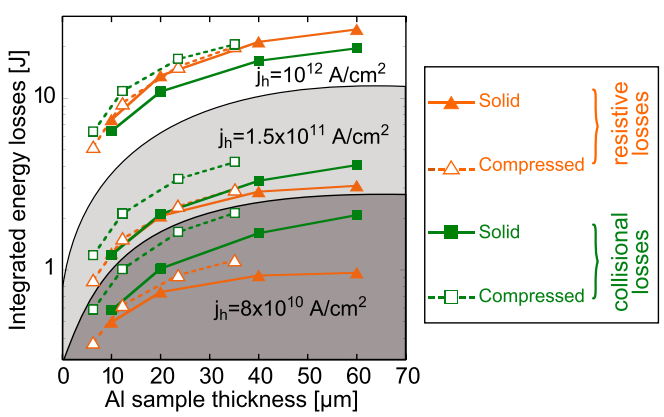

FIG. 5 (color online). Collisional (green squares) versus resistive (orange triangles) energy losses for solid (solid lines) and compressed (dashed lines) samples as a function of the crossed Al sample thickness. The gray areas represent the different SP-laser energies on target assumed in the simulations: $35 \mathrm{~J}$ (dark gray), $70 \mathrm{~J}$ (light gray) and $350 \mathrm{~J}$ (white). The laser-to-fast electron conversion efficiency is fixed at $40 \%$ in all cases.

this small current density, the resistive losses, only about a factor 2 lower, are not negligible in both solid and compressed Al. The correspondent resistive and collisional stopping powers averaged over the REB duration and over $35 \mu \mathrm{m}$ of crossed material are, respectively, $0.6-0.8 \mathrm{keV} / \mu \mathrm{m}$ and $1.1-1.5 \mathrm{keV} / \mu \mathrm{m}$, ranging from cold solid to warm and two times solid density Al.

For $j_{h}>10^{11} \mathrm{~A} / \mathrm{cm}^{2}$ (light gray background area) the resistive losses are at the same level as the collisional losses in solid Al. There is still a difference in resistive losses between solid and compressed samples, but only for a thickness greater than $20 \mu \mathrm{m}$. For $j_{h}>10^{12} \mathrm{~A} / \mathrm{cm}^{2}$ (white background area) such difference vanishes completely over the tested sample thickness. Indeed, at such high current densities, the characteristic electron plasma heating time up to the Fermi temperature, $\tau_{h} \sim$ $3 n_{e} T_{F} / 2 \eta j_{h}^{2} \approx 0.2$ ps is very short compared to the REB duration. Thus, the plasma is heated rapidly upon REB injection to temperatures where the resistivity is governed by the electron-electron collisions, independently of the initial temperature. It would then not be possible to differentiate the resistive from the collisional losses by comparing solid and compressed targets, as they will not depend on the plasma density.

Extending our measurements to FI scale, where $j_{h} \sim$ $10^{14} \mathrm{~A} / \mathrm{cm}^{2}$, we estimate that the dense hydrogen plasma will be heated in less than $10 \mathrm{fs}$ to temperatures of a few $100 \mathrm{eV}$, where the resistivity is as low as $10^{-9} \Omega \mathrm{m}$ [32]. Therefore, the resistive stopping power will be practically instantaneously reduced to the level of $\approx 1 \mathrm{keV} / \mu \mathrm{m}$, not undermining the core ignition.

In conclusion, absolute $K_{\alpha}$ experimental measurements, characterizing the REB transport, are in good agreement with a numerical hybrid model in cold-solid and in warmdense $\mathrm{Al}$ (produced by laser-driven shock compression), thanks to an improved description of the low energy part of the REB spectrum. Experimental and numerical results 
show higher collisional and resistive energy losses in compressed compared to solid $\mathrm{Al}$, due to higher density and initial resistivity, respectively. Although the collisions dominate in our experimental conditions, the resistive effects, responsible of one third of the total energy losses, are also significant. Hybrid transport simulations predict that when the REB current density exceeds $10^{11} \mathrm{~A} / \mathrm{cm}^{2}$ both energy loss mechanisms are comparable in solid Al. For $j_{h} \geq$ $10^{12} \mathrm{~A} / \mathrm{cm}^{2}$, they are comparable in warm-dense $\mathrm{Al}$ with density till few hundreds the critical density. Nonetheless, for very high densities, as the resistive energy losses are expected not to depend on the target density, due to the rapid heating of the background medium, the collisional losses will again be dominant. Though still restricted to underscaled ignition conditions, our measurements can be used for benchmarking models of fast electron beam transport, with a predictive capability for the full-scalled FI regime [33].

The authors acknowledge the French Agency for Research through project TERRE ANR-2011-BS04-014 in undertaking this work. We thankfully acknowledge LULI for giving access to the LULI2000 facility in the context of the preparatory phase of the HiPER project (Work Package 10: experimental validation program), as well as the support of the LULI2000 engineering staff in designing the setup and conducting the experiment. Alphanov is also gratefully acknowledged for the laser cutting of the targets. The numerical study was supported by Grant No. ENE2009-11668 of the Spanish Ministry of Education and Research and by the European Science Foundation SILMI program, and used HPC resources from CeSViMa and from GENCI-CINES (Grants No. 2011-056129 and No. 2012-056129). M.C. participated in this work thanks to funding from EPSRC (U.K.). The authors gratefully acknowledge L. Gremillet for fruitful discussions.

*santos.joao@celia.u-bordeaux1.fr

[1] M. Tabak, J. Hammer, M.E. Glinsky, W. L. Kruer, S. C. Wilks, J. Woodworth, E. M. Campbell, M. D. Perry, and R. J. Mason, Phys. Plasmas 1, 1626 (1994).

[2] R. Kodama et al., Nature (London) 412, 798 (2001).

[3] M. H. Key et al., Phys. Plasmas 5, 1966 (1998).

[4] P. M. Nilson, W. Theobald, J. Myatt, C. Stoeckl, M. Storm, O. V. Gotchev, J. D. Zuegel, R. Betti, D. D. Meyerhofer, and T. C. Sangster, Phys. Plasmas 15, 056308 (2008).

[5] B. Westover, C.D. Chen, P.K. Patel, M. H. Key, H. McLean, R. Stephens, and F. N. Beg, Phys. Plasmas 18, 063101 (2011).
[6] S. Atzeni, Phys. Plasmas 6, 3316 (1999).

[7] A. J. Kemp, Y. Sentoku, V. Sotnikov, and S.C. Wilks, Phys. Rev. Lett. 97, 235001 (2006).

[8] J.S. Green et al., Phys. Rev. Lett. 100, 015003 (2008).

[9] A. Debayle, J. J. Honrubia, E. d'Humières, and V.T. Tikhonchuk, Phys. Rev. E 82, 036405 (2010).

[10] L. Gremillet, G. Bonnaud, and F. Amiranoff, Phys. Plasmas 9, 941 (2002).

[11] M. Manclossi, J. Santos, D. Batani, J. Faure, A. Debayle, V. Tikhonchuk, and V. Malka, Phys. Rev. Lett. 96, 125002 (2006).

[12] J. J Honrubia and J. Meyer-ter-Vehn, Nucl. Fusion 46, L25 (2006).

[13] J. R. Davies, A. R. Bell, M. G. Haines, and S. M. Guerin, Phys. Rev. E 56, 7193 (1997).

[14] J. J. Honrubia, M. Kaluza, J. Schreiber, G. D. Tsakiris, and J. Meyer-ter-Vehn, Phys. Plasmas 12, 052708 (2005).

[15] E. Martinolli et al., Phys. Rev. E 73, 046402 (2006).

[16] J. J. Santos, A. Debayle, Ph. Nicolai, V. Tikhonchuk, M. Manclossi, D. Batani, A. Guemnie-Tafo, J. Faure, V. Malka, and J. J. Honrubia, Phys. Plasmas 14, 103107 (2007).

[17] T. A. Hall et al., Phys. Rev. Lett. 81, 1003 (1998).

[18] D. Batani et al., Phys. Rev. E 61, 5725 (2000).

[19] J. J. Santos et al., Plasma Phys. Controlled Fusion 51, 014005 (2009).

[20] J. J. Santos et al., J. Phys. Conf. Ser. 244, 022060 (2010).

[21] H. Nakamura, Y. Sentoku, T. Matsuoka, K. Kondo, M. Nakatsutsumi, T. Norimatsu, H. Shiraga, K. Tanaka, T. Yabuuchi, and R. Kodama, Phys. Rev. Lett. 100, 165001 (2008).

[22] F. Perez et al., Phys. Rev. Lett. 107, 065004 (2011).

[23] P.-H. Maire, R. Abgrall, J. Breil, and J. Ovadia, SIAM J. Sci. Comput. 29, 1781 (2007).

[24] M. N. Quinn et al., Plasma Phys. Controlled Fusion 53, 025007 (2011).

[25] K. Eidmann, J. Meyer-ter-Vehn, T. Schlegel, and S. Huller, Phys. Rev. E 62, 1202 (2000).

[26] B. Chimier, V. T. Tikhonchuk, and L. Hallo, Phys. Rev. B 75, 195124 (2007).

[27] R. B. Stephens et al., Phys. Rev. E 69, 066414 (2004).

[28] B. Vauzour et al., Nucl. Instrum. Methods Phys. Res., Sect. A 653, 176 (2011).

[29] J. F. Seely, L. T. Hudson, G. E. Holland, and A. Henins, Appl. Opt. 47, 2767 (2008).

[30] Y. Sentoku and A. J. Kemp, J. Comput. Phys. 227, 6846 (2008).

[31] C. Hombourger, J. Phys. B 31, 3693 (1998).

[32] S. Ichimaru and S. Tanaka, Phys. Rev. A 32, 1790 (1985).

[33] D. J. Strozzi, M. Tabak, D. J. Larson, L. Divol, A. J. Kemp, C. Bellei, M. M. Marinak, and M. H. Key, Phys. Plasmas 19, 072711 (2012). 